\title{
Tetrathionase: The Differential Effect of Temperature on Growth and Adaptation
}

\author{
BY R. KNOX \\ Public Health Laboratory, University of Oxford
}

SUMMARY: Cells of a tetrathionate-reducing coliform organism growing in semianaerobic conditions gained no advantage from the presence of tetrathionate at $41^{\circ}$, but at lower temperatures (e.g. $34^{\circ}$ ) they grew much better with than without it. When freely supplied with oxygen, the cells grew about as well at $41^{\circ}$ as at $34^{\circ}$. The adaptive formation of tetrathionase in washed suspension has already been shown to diminish with increase of temperature from $34^{\circ}$ to $44^{\circ}$, whereas the activity of the enzyme when formed increases with temperature in this range; nitratase, on the other hand, is still actively formed at temperatures as high as $44^{\circ}$. It is clear that whatever factors may be necessary for adaptation, one of them is more sensitive to heat than either nitratase formation or the overall growth process.

In previous work the following facts about the bacterial enzyme system tetrathionase were established:

(1) Salmonellae and a number of other Gram-negative bacteria can reduce tetrathionate quantitatively to thiosulphate (Knox, Gell \& Pollock, 1943). Both the activity of the fully developed enzyme and its adaptive formation can be demonstrated in washed suspension in the presence of a suitable $\mathbf{H}$ donator (Pollock \& Knox, 1943; Knox \& Pollock, 1944).

(2) The velocity of reduction by adapted cell suspensions increases with temperature at least up to $40^{\circ}$ or even higher. Adaptation, on the other hand, is almost completely suppressed at $41-42^{\circ}$, while it occurs rapidly at $37^{\circ}$ and even lower temperatures (Pollock, 1945).

(3) The reduction of tetrathionate is of value in growth to those organisms that can reduce it. Tetrathionate is a selective hydrogen acceptor and an alternative to oxygen; it is not so efficient as oxygen but it enables a broth culture to reach in a few hours a population two to three times the population reached in unaerated broth (Knox, 1945).

The relation between adaptation (which may involve the biosynthesis of a specific enzyme or enzyme system) and growth of a bacterial culture (synthesis of bacterial protoplasm as a whole) is one of intriguing interest. A study has now been made of the effect of temperature on the capacity of a bacterial culture to reduce tetrathionate during growth.

\section{METHODS}

The culture used was the coliform organism of intermediate type labelled '1433' (Pollock, 1946). Cells from an overnight broth culture prepared from stock agar slopes were heavily inoculated, usually so as to give just visible turbidity at the start, into $60-80 \%$ tryptic heart broth containing $0.05 \mathrm{M}$ phosphate at pH 7.6. Tetrathionate was used in a concentration of $0 \cdot 02 \mathrm{M}$. Cultures were incubated in thermostatically controlled water-baths at different temperatures, 
static cultures in 6 in. $\times \frac{3}{4}$ in. or 6 in. $\times \frac{5}{8}$ in. tubes, but aerated cultures in larger tubes through which oxygen was bubbled and to which a few drops of undecanol were added to suppress frothing (Linggood \& Fenton, 1947). Either the culture tubes themselves or suitable samples, diluted if necessary, were removed for estimation of turbidity in a Hilger Biochem absorptiometer. In some experiments total counts were performed using a Helber counting-chamber and dark-ground illumination, and viable counts by surface plating of suitable dilutions on to nutrient agar.

\section{RESULTS}

Fig. 1 illustrates the growth of organism 1433 in buffered broth at $34^{\circ}$ and $41^{\circ}$ under different conditions. In freely oxygenated broth growth at $41^{\circ}$ was at least as good as at $34^{\circ}$. There was some variation in different experiments as it

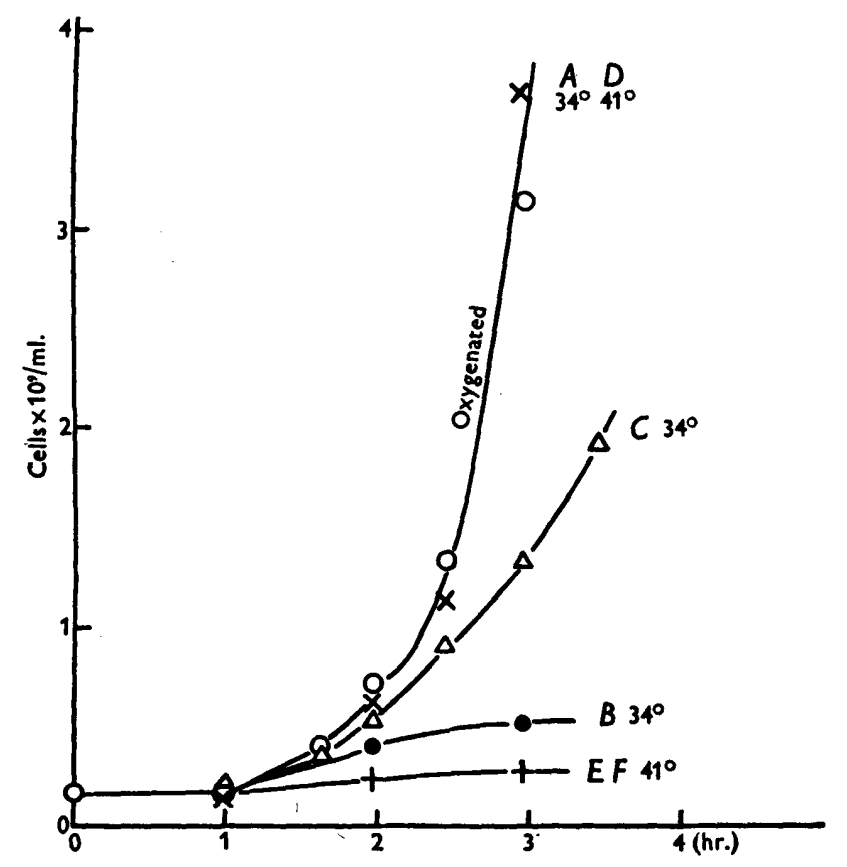

Fig. 1. Growth of organism 1433 in buffered broth at $34^{\circ}$ and $41^{\circ}$. At $34^{\circ}: A$, oxygenated; $B$, semi-anaerobic; $C$, tetrathionate $(0.02 \mathrm{M})$. At $41^{\circ}: D$, oxygenated; $E$, semi-anaerobic; $F$, tetrathionate $(0.02 \mathrm{M})$.

was difficult to maintain exactly equivalent oxygenation at the two temperatures. In some experiments growth at $41^{\circ}$ was initially a good deal faster than at $34^{\circ}$. In static broth cultures growth was poor at both temperatures as the limiting population was reached after only a few cell divisions, but growth at $41^{\circ}$ was slightly less than at $34^{\circ}$. In tetrathionate broth growth at $34^{\circ}$ was good, though the cultures never reached the same density as in oxygen, but at $41^{\circ}$ it was poor. These experiments showed that although with a free supply of $\mathrm{O}_{2}$ the cells grew equally well at $41^{\circ}$ and at $34^{\circ}$, it was only at the lower 
temperature that they gained an advantage from using tetrathionate as an alternative $\mathbf{H}$ acceptor to oxygen.

Measurement of tetrathionate reduction by the cells in this experiment at the two temperatures showed that at $84^{\circ}$ tetrathionate was rapidly reduced to thiosulphate, whereas at $41^{\circ}$ the amount reduced was negligible.

Fig. 2 shows for comparison the behaviour of adapted and unadapted cells of organism 1433 in washed suspension at the two temperatures used in the growth experiments. Reduction of tetrathionate at $41^{\circ}$ was, as expected, faster than at $34^{\circ}$, whereas adaptation by unadapted cells was much faster at $34^{\circ}$ than at $41^{\circ}$, although not completely suppressed at the higher temperature.

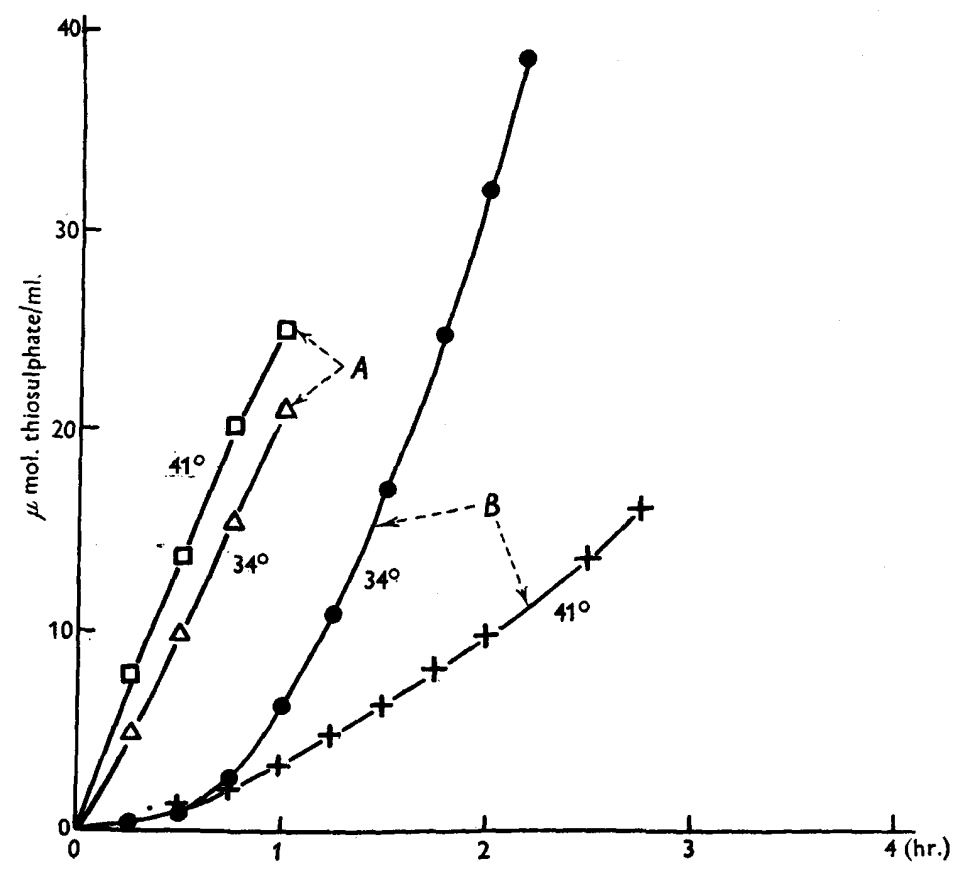

Fig. 2. Reduction of tetrathionate by washed suspensions of organism 1433 at $34^{\circ}$ and $41^{\circ}$. $A$, adapted cells ; $B$, unadapted cells.

It is not certain that adaptation during growth is the same process as adaptation in washed suspension, but it is interesting to observe that the known temperature-sensitivity of the adaptive mechanism in washed suspension is paralleled by the behaviour of the same organism during what may be described as 'adaptive' growth. It seems that adaptation is a necessary preliminary for adequate growth in semi-anaerobic conditions-since adequate growth occurs, even in a rich medium, only at temperatures low enough to leave undamaged the mechanisms of adaptation. There was found to be a fairly wide temperature optimum for 'adaptive' growth, between $\mathbf{3 2}^{\circ}$ and $\mathbf{3 8}^{\circ}$. Above this range 'adaptive' growth still occurred at $40^{\circ}$ but declined very sharply between $40^{\circ}$ and $41^{\circ}$, until at $42^{\circ}$ it was completely suppressed. 
In view of the known selective value of tetrathionate, a few experiments were performed with mixed cultures of the tetrathionate reducer 1433 and a strain of Bact. coli which did not reduce tetrathionate. Tubes of heart broth and of tetrathionate heart broth, containing mixtures of the two organisms in about equal numbers, were incubated at $34^{\circ}$ and $41^{\circ}$. The heart-broth tubes were freely oxygenated throughout the experiments. The results, as might be expected, were erratic; but in one experiment whereas the proportion of reducers to non-reducers either remained unchanged or decreased in oxygen at $34^{\circ}$ and $41^{\circ}$ and in tetrathionate at $41^{\circ}$, it increased in tetrathionate at $34^{\circ}$, showing that the reducer gained an advantage over the non-reducer in the presence of tetrathionate only when growing at a temperature at which adaptation and therefore utilization of the tetrathionate occurred.

The failure of organism 1433 to reduce tetrathionate during growth at $41^{\circ}$ was presumably due to a noxious effect of temperature, and perhaps of tetrathionate as well, on some metabolic process in the cells. The least sensitive mechanism is almost certainly not the tetrathionase enzyme system, since in washed suspension this is more active at $41^{\circ}$ than at $34^{\circ}$. Nor is it likely that the growth of the whole bacterial cells is much affected, since freely aerated cultures of organism 1433 grow as well at $41^{\circ}$ as at $34^{\circ}$, not only in the absence, but also in the presence of tetrathionate. All the evidence suggests that it is the process of adaptation as opposed to the overall growth process that is differentially and selectively interfered with by the increase of temperature.

To what extent this inhibition of adaptation can itself be explained by an increased toxic effect of tetrathionate at higher temperatures is not clear. At this point some experiments with nitratase are relevant.

Cultures of organism 1433 were incubated in the usual way at different temperatures, but with nitrate instead of tetrathionate. At $41^{\circ}$, whereas growth in tetrathionate broth was very poor, in nitrate broth it was as good as at $34^{\circ}$, and nitrate still stimulated growth up to $42-43^{\circ}$. Even the presence of tetrathionate in addition only partially suppressed this stimulatory effect. These experiments suggested that nitratase adaptation is less thermolabile than tetrathionase adaptation. This has been further demonstrated by direct experiments on the effect of temperature on the two adaptive processes in washed suspension. A considerable adaptive production of nitratase occurred even at $44.5^{\circ}$, whereas tetrathionase adaptation was almost abolished at $42^{\circ}$ and considerably inhibited at $40^{\circ}$ (Pollock \& Wainwright, unpublished observations). Elsewhere we have shown (Jebb, Knox \& Tomlinson, 1950) that tetrathionate concentrations down to $0.005 \mathrm{M}$ do have some inhibitory effect on adaptation; but since the effect of tetrathionate on 'adaptive' growth in nitrate is not great even at $42^{\circ}$ it seems unlikely that tetrathionate should suppress the formation of 'its own' enzyme even more than that of another. Finally, it was found in growth experiments in the presence of tetrathionate at $3_{4}^{\circ}$ and $42^{\circ}$, that ' adaptive' growth still failed to occur at the higher temperature even when the tubes contained as little as $0.0025 \mathrm{M}$ tetrathionate. 


\section{DISCUSSION}

If tetrathionate is to give an effective stimulus to the growth of cells which can use it as a hydrogen acceptor when oxygen is limited, it is clear that growth must occur at a temperature at which adaptation can take place, namely, at around $32-37^{\circ}$. At higher temperatures, appreciable growth in a medium in which tetrathionate is the only effective hydrogen acceptor fails because adaptation is suppressed. This suppression may in turn be merely a reflexion of the fact that tetrathionate is a somewhat toxic substance whose toxicity might be expected to increase with rise in temperature. But whatever the explanation, it is evident that here is an example of a specific adaptation process being almost completely suppressed at a temperature at which growth seems quite unimpaired. The reverse might perhaps have been expected, since increase in bacterial protoplasm (growth) is presumably the result of the co-ordinated working of many enzyme systems, and the optimum temperature for growth no doubt depends on a balance resulting from the temperature optima of many of these systems, the most heat-sensitive of which might be expected to be growth-limiting. With tetrathionase it seems that while this enzyme system when formed is less sensitive, the actual process of its formation is much more sensitive to increase in temperature than is the growth process as a whole. There seems to be little information as to the heat-sensitivity of other adaptive enzymes. It is interesting that nitratase adaptation seems to be much less heatsensitive than tetrathionase; this is further evidence of the comparative independence of these two adaptation processes.

It is only in recent years that the importance of adaptation in bacterial growth has been appreciated, and an investigation into the temperature optima of different adaptive systems may be expected to add considerably to our understanding of the growth processes of the bacterial cell.

My thanks are due to Dr M. R. Pollock and Dr S. D. Wainwright for the experimental data referred to in the text.

\section{REFERENCES}

JEBB, W. H. H., Knox, R. \& Tombinson, A. H. (1950). Some factors influencing the rate of formation of tetrathionase. J. gen. Microbiol. 4, 374.

KNox, R. (1945). The effect of tetrathionate on bacterial growth. Brit. J. exp. Path. 26, 146.

Knox, R., Gell, P. G. H. \& Pollock, M. R. (1943). The selective action of tetrathionate in bacteriological media. J. Hyg., Camb., 43, 147.

Knox, R. \& Pollock, M. R. (1944). Bacterial tetrathionase: Adaptation without demonstrable cell growth. Biochem. J. 38, 299.

LingGood, F. V. \& Fenton, E. L. (1947). The production of diphtheria toxin by submerged culture in shaking flasks. Brit. J. exp. Path. 28, 354.

Pollock, M. R. (1945). The influence of temperature on the adaptation of 'tetrathionase' in washed suspensions of Bact. paratyphosum B. Brit. J. exp. Path. 26, 410.

Pollock, M. R. (1946). Adaptation of 'nitratase' in washed suspensions of bacteria. Brit. J. exp. Path. 27, 419.

Pollock, M. R. \& KNox, R. (1943). Bacterial reduction of tetrathionate. Biochem.J. 37, 476 .

(Received 5 December 1949) 